\title{
Early coevolution of life and the geodynamo suggested by potential $\sim 3.4$ billion-year-old magnetofossils
}

\author{
JOHN TARDUNO ${ }^{1,2}$, AXEL HOFMANN ${ }^{3}$, RORY COTTRELL ${ }^{1}$ \\ ${ }^{1}$ University of Rochester, Department of Earth \& \\ Environemntal Sciences, Rochester, NY 14627, United \\ States, john.tarduno@rochester.edu \\ ${ }^{2}$ University of Rochester, Department of Physics \& \\ Astronomy, Rochester, NY, 14627, United States \\ ${ }^{3}$ University of Johannesburg, Department of Geology, \\ Johannesburg, South Africa
}

The geomagnetic field is important for our understanding of the evolution of life because of its role in shielding Earth from intense solar winds emanating from the young Sun. A relatively strong Paleoarchean to Hadean geomagnetic field recorded from single crystal paleointensity studies (Tarduno et al., Science, 2010, 2015; Tarduno et al., PNAS, 2020) suggests that shielding, crucial for the preservation of habitability, was in place very early in the history of the planet. But this also implies that bacteria could have used the geomagnetic field for orientation and motion billions of years before the oldest reported putative magnetofossils. Here we use multiple approaches, including rock magnetism, electron microscopy, and ferromagnetic resonance, to test whether fossil bacterial magnetite is present in micro-paleo-redox environments of the $\sim 3.40$ Ga Buck Reef Chert (Barberton Greenstone Belt, South Africa). Magnetic hysteresis properties indicate a magnetic particle assemblage that is a mixture of domain states, with one component indicative of the single domain behavior that characterizes bacterial magnetite. Moreover, electron microscopy of magnetic separates and in-situ particles reveals the presence of cubooctahedral to quasi-rectangular and hexagonally shaped grains that also are typical of biogenic magnetite. In addition, ferromagnetic resonance spectra collected on bulk samples suggest a magnetic anisotropy similar to that observed in spectra collected on some modern strains of magnetotactic bacteria. These observations support the presence of fossil bacterial magnetite in the Buck Reef Chert and, together with paleointensity data, suggest an early coevolution of magnetotactic bacteria and the geodynamo. 